\title{
Modelo explicativo de la satisfacción del profesorado en el periodo de enseñanza remota en México
}

\section{Explanatory model of teacher satisfaction in the remote teaching period in Mexico}

Óscar Reynoso.

Centro Universitario de los Altos de la Universidad de Guadalajara (México). ulises.reynoso@academicos.udg.mx

Samuel Portillo. Instituto Tecnológico de Sonora (México). samuelport90@gmail.com

Lidia Castellanos. Instituto Tecnológico de Sonora (México). lidiaisabelcp@yahoo.es

\section{RESUMEN.}

El presente trabajo tuvo como principal objetivo conocer el grado de satisfacción del profesorado de Educación Básica, así como los factores asociados a la misma, con respecto a los resultados obtenidos durante el período de enseñanza remota de emergencia ocasionado por la pandemia de Covid-19. Se trata de un estudio de carácter explicativo, para el cual se consideró una muestra seleccionada por conveniencia integrada por un total de 1844 docentes de tres estados de la República Mexicana. Para la recolección de la información se utilizó un cuestionario ad hoc que consideró como dimensiones de análisis las siguientes: dispositivos y conectividad, apoyo familiar e institucional, preparación y capacitación. Para el procesamiento de los datos se trabajó con el programa SPSS versión 21. El análisis de los resultados se desarrolló a partir del uso de pruebas de hipótesis no paramétricas específicamente la $H$ de Krskal Wallis y el coeficiente Rho de Spearman además de un análisis de regresión lineal multivariada que permitió generar tres modelos explicativos de la satisfacción docente durante el período de enseñanza remota, uno para cada nivel escolar considerado en el estudio. Los resultados demuestran que los niveles de satisfacción aumentan al incrementarse la disposición de dispositivos y recursos tecnológicos, el apoyo familiar e institucional y la preparación y capacitación. Asimismo, se hace referencia a una mayor satisfacción del profesorado en los niveles de educación preescolar y primaria en comparación de quienes laboran en educación secundaria.

\section{PALABRAS CLAVE.}

Satisfacción docente, enseñanza remota, COVID-19.

\section{ABSTRACT.}

This paper had as main objective to know the degree of satisfaction of the Basic Education teachers, as well as the factors associated with it, with regard to the results obtained during the remote emergency teaching period caused by the Covid-19 pandemic. This is an explanatory study, for which a sample of 1844 teachers from three Mexican states was

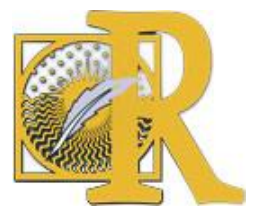


selected for convenience. The information was collected using an ad hoc questionnaire that considered the following dimensions of analysis: devices and connectivity, family and institutional support, preparation and training. The analysis of the results was carried out using non-parametric hypothesis tests, specifically the $\mathrm{H}$ of Krskal Wallis and the Rho coefficient of Spearman, in addition to a multivariate linear regression analysis that made it possible to generate three explanatory models of teacher satisfaction during the period of remote education, one for each school level considered in the study. The results show that satisfaction levels increase as the availability of technological devices and resources, family and institutional support, and preparation and training increase. Reference is also made to greater teacher satisfaction at the preschool and primary education levels compared to those working in secondary education.

\section{KEY WORDS.}

Teacher satisfaction, remote teaching, COVID-19.

\section{Introducción.}

El actual estado de emergencia sanitaria provocado por el virus SARS-CoV2 y su enfermedad Covid-19, ha propiciado la paralización de actividades escolares presenciales entodos los niveles educativos de manera indefinida. Ello, ha traído como consecuencia replanteamientos trascendentales en los procesos de enseñanza aprendizaje a realizar durante el confinamiento, los cuales han optado por transitar de un sistema tradicional presencial a otro completamente virtual. Dicho proceso integra la idea de llevar educación a los hogares a través de educación a distancia en modalidad remota, a través del uso de tecnología con fines educativos (Álvarez, Gardyn, lardelevsky y Rebello, 2020; Arce-Peralta, 2020; Archer y De Gracia, 2020; Bonilla-Guachamín, 2020; Cóndor-Herrera, 2020; Fanelli, Marquina y Rabossi, 2020; Figallo, 2020; Morales, 2020;Pérez-Narváez y Tufiño, 2020; Ruiz, 2020; UNESCO, 2020; Vivanco-Saraguro, 2020).

El cambio hacia una transición educativa virtual, ha repercutido en el profesorado de manera directa, ya que habrá de utilizar los dispositivos tecnológicos a su alcance para enseñar, además de realizar cambios sustantivos a su práctica docente, teniendo que adaptar sus planeaciones, tiempos, estrategias de enseñanza y los contenidos al entorno digital. Ello, en un contexto en el que se exige responder con prontitud, en detrimento de la calidad por el corto tiempo de actuación, sumado a las múltiples dificultades que presentan los padres de familia y el estudiantado en sus hogares en cuanto al acceso a tecnología, conectividad, tiempo compartido de uso de dispositivos, dificultades para el estudio independiente, carencia de apoyo y orientación en las actividades escolares, entre otro tipo de problemáticas adversas al logro de los objetivos educativos y que se configuran como brechas educativas actuales (Archer y De Gracia, 2020; Arriagada, 2020; Bocchio, 2020; Cifuentes-Faura, 2020; Fernández Enguita, 2020; Malo, Maldonado-Maldonado, Gacel y Marmolejo, 2020; Murillo y Duk, 2020; Pardo y Cobo, 2020).

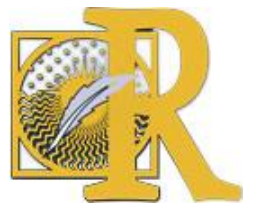

Fecha de recepción: 17-08-2020 Fecha de aceptación: 26-08-2020 
Si bien, los estudios en el ámbito educativo laboral indican que realizar actividades de docencia representa una fuente de satisfacción (Cladellas-Pros, Castelló-Tarrida y ParradoRomero, 2018) realizarla en las condiciones actuales (en contexto de crisis sanitaria y mediada por tecnología) quizá no lo resulte. En este sentido, es necesario analizar las valoraciones de satisfacción e insatisfacción hacia la práctica docente que realiza el profesorado como producto de la buena o mala calidad de la enseñanza durante la fase de confinamiento (Sonlleva, Martínez y Monjas, 2019). Por lo tanto, resulta de suma importancia visualizar los elementos que motivan al profesorado y causan satisfacción en los procesos de enseñanza remota que se han venido realizando durante la fase de distanciamiento social para elevar el desempeño y calidad de enseñanza durante la práctica.

Como referente teórico, la satisfacción docente se ha visualizado como la manifestación de una actitud general ante el trabajo (Caballero, 2002; Cantón y Téllez, 2016;Guerrero, Parra y Arce, 2018; Oros y Main, 2004), el estado emocional positivo o el resultado de la valoración que el individuo hace de su trabajo o de sus experiencias con el mismo (Locke, 1976), el resultado de la comparación entre lo que el docente es y las expectativas de lo que debería ser (Limaymanta-Álvarez, 2019), el producto de las condiciones materiales reales y concretas (Ureña y Castro, 2009) así como las condiciones psicológicas de los integrantes del grupo o colectivo laboral (Muñoz-Chávez y López-Chau, 2018) y el grado motivacional de los sujetos orientado al logro de objetivos (Franco-López, López-Arellano y Arango-Botero, 2019), así como un estado de bienestar general (Alves, Lopes y Precioso, 2020).

Para efectos del presente trabajo, la satisfacción del docente será entendida como el criterio de evaluación que integra actitudes y emociones positivas hacia el trabajo para el aseguramiento de la calidad y los resultados educativos obtenidos durante el proceso de enseñanza/aprendizaje. En este sentido, el profesorado que manifiesta satisfacción con sus labores educativas, será aquel que profesionalmente valide la calidad de los resultados en cuanto al trabajo que se realiza, manifieste preparación, goce, disfrute y se sienta apoyado y valorado al momento de realizar sus actividades escolares. Caso contrario, será la insatisfacción docente, visualizada como la integración de experiencias negativas que generan malestar hacia la labor, ocasionadas por estrés, ansiedad y sentimientos de frustración ligados al detrimento de la calidad y resultados educativos.

Considerando lo anterior, se presenta un análisis explicativo derivado de los resultados escolares obtenidos durante la fase de confinamiento por niveles educativos (preescolar, primaria y secundaria) a fin de descubrir en qué medida las dimensiones de dispositivos y conectividad, apoyo familiar e institucional y la preparación y capacitación se encuentran relacionados y permiten explicar la satisfacción del profesorado durante el período de enseñanza remota.

\section{Método.}

El estudio se desarrolló bajo el enfoque cuantitativo de investigación, mediante un diseño observacional, transversal y de alcance explicativo (Hernández y Mendoza, 2018). La muestra se conformó por 1844 docentes provenientes de tres estados de la República Mexicana (Aguascalientes, Sonora y Tlaxcala) y, en su mayoría, de contextos urbanos o suburbanos (90.1\%). El $77.3 \%$ (1426) eran mujeres y el $22.7 \%$ hombres (418), con un

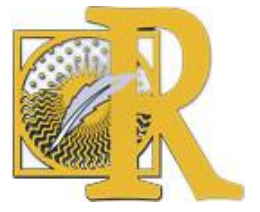

Fecha de recepción: 17-08-2020 Fecha de aceptación: 26-08-2020

Reynoso, O., Portillo, S. \& Castellanos, L. (2021). Modelo explicativo de la satisfacción del profesorado en el periodo de enseñanza remota en México

International Journal of Educational Research and Innovation (IJERI), 15, 229-247

ISSN: 2386-4303 DOI https://doi.org/10.46661/ijeri.5236 
promedio de edad de 40 años ( $\mathrm{DE}=9.742$ ). En relación con su práctica, el $16.2 \%$ se desempeñaba en el nivel preescolar, el $72.1 \%$ en primaria y el $11.7 \%$ en secundaria, tanto de instituciones públicas (88.2\%) como privadas (11.8\%). Finalmente, en cuestión de su experiencia laboral, el $15.7 \%$ tenía menos de cinco años de servicio, el $26.1 \%$ entre cinco y diez años, y el $58.1 \%$ contaba con más de 10 años de experiencia en la docencia. Los participantes fueron seleccionados a través de un muestreo por conveniencia.

\subsection{Instrumentos.}

Se utilizó un cuestionario desarrollado por el equipo de investigación que contenía 17 reactivos agrupados en cuatro temáticas: Satisfacción docente (que abordaba el agrado por los resultados obtenidos durante el periodo de enseñanza remota), Dispositivos y conectividad (aludiendo a la disposición de los recursos tecnológicos necesarios para efectuar su labor a distancia), Apoyo familiar e institucional (respecto a la percepción de soporte recibido de parte de sus centros de trabajo y las familias de los estudiantes) y Preparación y capacitación (sobre las acciones emprendidas y disposiciones para enfrentar la enseñanza remota). En todos los casos, el formato de respuesta era de escalamiento ordinal tipo Likert, en donde 1 representaba Nada y 5Totalmente. Si bien el instrumento pretendía obtener puntuaciones individuales y no factoriales o dimensionales por cada temática, se constató su confiabilidad mediante el coeficiente de consistencia Alfa de Cronbach $(\alpha=.874)$.

\subsection{Procedimiento.}

Para la fase de recolección de datos, se utilizó la herramienta de formularios de Google para la aplicación del cuestionario, atendiendo a los protocolos de distanciamiento, así como para agilizar el tiempo de respuesta por medio de los dispositivos móviles, contando con el apoyo de la estructura educativa de Educación Básica en los estados de Aguascalientes, Sonora y Tlaxcala para su difusión y envío. El momento de recogida de datos se realizó durante el mes de julio, derivado del análisis de resultados obtenidos al cierre del ciclo escolar 2019-2020. El criterio de selección fue que los participantes realizaran actividades docentes, previa aceptación de participación voluntaria e informada del uso de datos con fines académicos. El formato de respuesta fue totalmente anónimo, sin alguna compensación a cambio. El tiempo de respuesta estimado para el cuestionario fue de 10 minutos.

\subsection{Análisis de datos.}

En primera instancia se utilizaron estadísticos descriptivos para identificar los niveles de respuesta en cada uno de los elementos del instrumento. Posteriormente, los resultados fueron comparados y correlacionados de acuerdo con nivel educativo de los docentes. Antes de ejecutar el análisis se revisó la distribución de los reactivos y se constató que no todos tenían una distribución normal. Debido a ello, se utilizó en ambos procedimientos pruebas de hipótesis no paramétricas, específicamente la $H$ de Kruskal Wallis en la comparación y el coeficiente Rho de Spearman para la correlación. Por último, se realizó un análisis de regresión lineal múltiple para identificar los elementos que explican en mayor medida la satisfacción docente. Se ejecutaron tres análisis distintos, uno para cada nivel educativo. Es

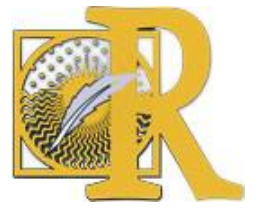

Fecha de recepción: 17-08-2020 Fecha de aceptación: 26-08-2020

Reynoso, O., Portillo, S. \& Castellanos, L. (2021). Modelo explicativo de la satisfacción del profesorado en el periodo de enseñanza remota en México

International Journal of Educational Research and Innovation (IJERI), 15, 229-247

ISSN: 2386-4303 DOI https://doi.org/10.46661/ijeri.5236 
preciso señalar que se confirmaron los supuestos necesarios para su ejecución, tanto a nivel de los predictores (revisión de la linealidad con la variable endógena y la no colinealidad entre ellas) así como en los residuos (normalidad, homocedasticidad e independencia) (Pardo y Ruiz, 2005).

\section{Resultados.}

Para dar orden a la presentación de resultados, en primera instancia se presentan los estadísticos descriptivos, tanto de forma general como por niveles educativos. Además de interpretar los promedios, se realizó una comparación para detectar diferencias estadísticamente significativas entre los tres grupos (preescolar, primaria y secundaria). La Tabla 1 muestra tales hallazgos.

Tabla 1 - Resultados generales y por nivel educativo

\begin{tabular}{lllllll}
\hline \multicolumn{1}{c}{ Reactivos } & Total & Preescolar & Primaria & Secundaria & $X$ & $p$ \\
\hline \multicolumn{1}{c}{ Satisfacción docente } & & & & & & \\
\hline $\begin{array}{l}\text { Satisfacción con los resultados } \\
\text { escolares obtenidos durante el }\end{array}$ & 3.29 & 3.36 & 3.28 & 3.27 & 2.489 & .115 \\
período de enseñanza remota & & & & & & \\
\hline Dispositivos y conectividad & & & & & & \\
\hline $\begin{array}{l}\text { Disponer de internet de banda ancha } \\
\text { o conexión a internet en el hogar } \\
\text { adecuada }\end{array}$ & 3.70 & 3.73 & 3.68 & 3.77 & .323 & .570 \\
\hline $\begin{array}{l}\text { Disponer de los dispositivos y recursos } \\
\text { tecnológicos necesarios }\end{array}$ & 3.83 & 3.90 & 3.79 & 3.99 & 1.843 & .175 \\
\hline
\end{tabular}

\section{Apoyo familiar e institucional}

\begin{tabular}{|c|c|c|c|c|c|c|}
\hline $\begin{array}{l}\text { La participación y apoyo de las } \\
\text { familias de los/las estudiantes }\end{array}$ & 3.25 & 3.16 & 3.30 & 3.02 & 5.986 & $.014^{*}$ \\
\hline $\begin{array}{l}\text { Apoyo institucional mediante la } \\
\text { habilitaron softwares } y / o \text { plataformas }\end{array}$ & 2.67 & 2.59 & 2.66 & 2.87 & .304 & .581 \\
\hline $\begin{array}{lc}\text { Apoyo institucional } \\
\text { materiales } & \text { de } \\
\text { digitalizados/impresos }\end{array}$ & 3.03 & 3.16 & 3.01 & 2.91 & 2.102 & .147 \\
\hline
\end{tabular}

\begin{tabular}{llllllll}
\hline $\begin{array}{l}\text { Apoyo institucional mediante la } \\
\text { elaboración de guías para orientar el } \\
\text { trabajo del colectivo docente }\end{array}$ & 3.33 & 3.56 & 3.30 & 3.21 & 8.106 & $.004^{* *}$ \\
\hline $\begin{array}{l}\text { Apoyo institucional } \\
\text { dispositivos tecnológicos }\end{array}$ & mediante & 1.95 & 1.96 & 1.94 & 2.02 & .172 & .678 \\
\hline Apoyo institucional general & 3.66 & 3.73 & 3.64 & 3.68 & .954 & .329 \\
\hline
\end{tabular}


Óscar Reynoso, Samuel Portillo \& Lidia Castellanos. Modelo explicativo de la satisfacción del profesorado en el periodo de enseñanza remota en México

\begin{tabular}{lllllllll}
$\begin{array}{l}\text { Adecuada } \\
\text { superiores }\end{array}$ & comunicación con 4.50 & 4.58 & 4.49 & 4.39 & 1.700 & .192 \\
\hline
\end{tabular}

Preparación y capacitación

\begin{tabular}{|c|c|c|c|c|c|c|}
\hline $\begin{array}{l}\text { Preparación para realizar los cambios } \\
\text { requeridos }\end{array}$ & 3.84 & 3.89 & 3.82 & 3.84 & 1.075 & .300 \\
\hline $\begin{array}{l}\text { Preparación y disposición para } \\
\text { trabajar en colaboración con los/las } \\
\text { estudiantes y padres de familia }\end{array}$ & 3.99 & 4.05 & 3.97 & 4.07 & 1.692 & .193 \\
\hline $\begin{array}{l}\text { Capacitación mediante cursos y } \\
\text { talleres virtuales sobre el uso de } \\
\text { tecnología }\end{array}$ & 3.94 & 4.05 & 3.94 & 3.82 & 2.412 & .120 \\
\hline $\begin{array}{l}\text { Capacitación mediante tutoriales y } \\
\text { otros recursos en internet }\end{array}$ & 4.18 & 4.17 & 4.17 & 4.21 & .024 & .878 \\
\hline $\begin{array}{l}\text { Capacitación mediante la consultado a } \\
\text { otros profesionales y colegas de la } \\
\text { educación }\end{array}$ & 4.09 & 4.11 & 4.09 & 4.06 & .132 & .716 \\
\hline $\begin{array}{l}\text { Capacitación mediante los directivos } \\
\text { de las instituciones }\end{array}$ & 3.57 & 3.69 & 3.56 & 3.47 & 1.877 & .171 \\
\hline $\begin{array}{l}\text { Dominio de herramientas de } \\
\text { comunicación remota (Teams, Skype, } \\
\text { Zoom, Weber, Google Hangout, } \\
\text { Google Meet, JitsiMeet }\end{array}$ & 3.45 & 3.43 & 3.44 & 3.60 & .010 & .920 \\
\hline
\end{tabular}

* $p<.05 ;{ }^{* *} p<.05$

Fuente: Elaboración propia

Considerando que las respuestas de los participantes oscilaban en un rango de cuatro puntos (de 1 a 5), se realizó una segmentación para clasificar la puntuación en tres niveles: bajo (de 1 a 2.65), medio (de 2.66 a 3.65) y alto (de 3.66 a 5). Comenzando por la primera dimensión del instrumento, el profesorado de los tres niveles educativos manifestó un nivel medio de satisfacción con los resultados escolares obtenidos durante el período de enseñanza remota, sin diferencias significativas entre ellos. Enseguida, en el factor de Dispositivos y conectividad, los profesores manifestaron puntuaciones altas, tanto en el acceso a internet como en la disposición de los dispositivos y recursos tecnológicos necesarios para su práctica.

Al abordar el área del Apoyo familiar e institucional, se encontraron diferencias significativas entre los niveles educativos en la participación y apoyo de las familias de los/las estudiantes $(H=8.106 ; p=.014)$. Si bien los puntajes de los tres grupos fueron de nivel medio, los docentes de secundaria reportaron niveles inferiores de involucramiento familiar. Por su parte, el apoyo institucional presentó puntuaciones bastante desiguales. Mientras que algunos elementos como el apoyo mediante la elaboración de guías (en donde los docentes de preescolar

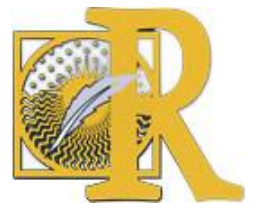

Fecha de recepción: 17-08-2020 Fecha de aceptación: 26-08-2020 
obtuvieron puntuaciones significativamente más altas $[H=8.106 ; p=.014]$ ), materiales de trabajo digitalizados/impresos y la habilitación de softwares y/o plataformas obtuvieron en su mayoría puntuaciones en el rango medio, el apoyo mediante dispositivos tecnológicos se mostró en niveles sumamente bajos. En cambio, fue la comunicación con los superiores donde se reportaron los niveles más altos. De forma general, las puntuaciones de apoyo institucional se ubicaron entre los límites de los niveles medio y alto. Finalmente, en la dimensión de Preparación y capacitación se presentaron niveles altos en la capacitación mediante tutoriales, consulta a otros profesionales de la educación y a través de cursos y talleres virtuales sobre el uso de tecnología. También la preparación para realizar los cambios requeridos durante el periodo de emergencia y la disposición para trabajar en colaboración con los/las estudiantes y padres de familia obtuvieron niveles altos. En este caso, sólo la capacitación y apoyo por parte de los directivos de las instituciones y el dominio de herramientas de comunicación remota mostraron niveles medios.

Continuando con el análisis de datos, se atendió la relación de cada una de las dimensiones con el factor de satisfacción. Dada la ausencia de la normalidad en la distribución de algunas de las preguntas, se utilizó el coeficiente de correlación de Spearman en la ejecución de dicha tarea. La Tabla 2 muestra los resultados de forma general y por nivel educativo.

Tabla 2 - Correlaciones con la satisfacción docente por nivel educativo

\begin{tabular}{|c|c|c|c|c|}
\hline \multirow[t]{2}{*}{ Reactivos } & \multicolumn{4}{|c|}{ Satisfacción docente } \\
\hline & Total & Preescolar & Primaria & Secundaria \\
\hline \multicolumn{5}{|l|}{ Dispositivos y conectividad } \\
\hline $\begin{array}{l}\text { Disponer de internet de banda ancha } 0 \\
\text { conexión a internet en el hogar adecuada }\end{array}$ & $.188^{\star *}$ & .100 & $.207^{\star *}$ & $.190^{\star *}$ \\
\hline $\begin{array}{l}\text { Disponer de los dispositivos y recursos } \\
\text { tecnológicos necesarios }\end{array}$ & $.257^{\star \star}$ & $.174^{\star *}$ & $.274^{\star *}$ & $.260^{\star *}$ \\
\hline \multicolumn{5}{|l|}{ Apoyo familiar e institucional } \\
\hline $\begin{array}{l}\text { La participación y apoyo de las familias de } \\
\text { los/las estudiantes }\end{array}$ & $.400^{\star *}$ & $.422^{\star *}$ & $.427^{\star \star}$ & $.255^{\star *}$ \\
\hline $\begin{array}{l}\text { Apoyo institucional mediante la habilitaron } \\
\text { softwares y/o plataformas }\end{array}$ & $.288^{\star *}$ & $.259^{* *}$ & $.286^{\star *}$ & $.343^{* *}$ \\
\hline $\begin{array}{l}\text { Apoyo institucional mediante materiales de } \\
\text { trabajo digitalizados/impresos }\end{array}$ & $.292^{\star *}$ & $.285^{\star *}$ & $.285^{\star *}$ & $.343^{\star \star}$ \\
\hline $\begin{array}{l}\text { Apoyo institucional mediante la elaboración } \\
\text { de guías para orientar el trabajo del colectivo } \\
\text { docente }\end{array}$ & $.299^{\star *}$ & $.337^{\star *}$ & $.289^{\star *}$ & $.303^{\star *}$ \\
\hline $\begin{array}{l}\text { Apoyo institucional mediante dispositivos } \\
\text { tecnológicos }\end{array}$ & $.246^{* *}$ & $.257^{\star *}$ & $.247^{\star *}$ & $.224^{* *}$ \\
\hline
\end{tabular}

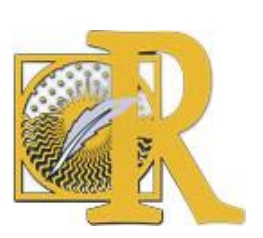


Óscar Reynoso, Samuel Portillo \& Lidia Castellanos. Modelo explicativo de la satisfacción del profesorado en el periodo de enseñanza remota en México

\begin{tabular}{lllll} 
Apoyo institucional general & $.358^{* *}$ & $.390^{* *}$ & $.356^{* *}$ & $.328^{* *}$ \\
\hline Adecuada comunicación con superiores & $.235^{* *}$ & $.180^{* *}$ & $.226^{* *}$ & $.349^{* *}$ \\
\hline \multicolumn{1}{c}{ Preparación y capacitación } & & & & \\
\hline $\begin{array}{l}\text { Preparación para realizar los cambios } \\
\text { requeridos }\end{array}$ & $.380^{* *}$ & $.376^{* *}$ & $.384^{* *}$ & $.357^{* *}$ \\
\hline $\begin{array}{l}\text { Preparación y disposición para trabajar en } \\
\text { colaboración con los/las estudiantes y padres } \\
\text { de familia }\end{array}$ & $.364^{* *}$ & $.342^{* *}$ & $.362^{* *}$ & $.393^{* *}$ \\
\hline $\begin{array}{l}\text { Capacitación mediante cursos y talleres } \\
\text { virtuales sobre el uso de tecnología }\end{array}$ & $.205^{* *}$ & 0.097 & $.202^{* *}$ & $.374^{* *}$ \\
\hline $\begin{array}{l}\text { Capacitación mediante tutoriales y otros } \\
\text { recursos en internet }\end{array}$ & $.228^{* *}$ & $.120^{*}$ & $.247^{* *}$ & $.263^{* *}$ \\
\hline $\begin{array}{l}\text { Capacitación mediante la consultado a otros } \\
\text { profesionales y colegas de la educación }\end{array}$ & $.217^{* *}$ & $.151^{* *}$ & $.235^{* *}$ & $.207^{* *}$ \\
\hline $\begin{array}{l}\text { Capacitación mediante los directivos de las } \\
\text { instituciones }\end{array}$ & $.297^{* *}$ & $.272^{* *}$ & $.278^{* *}$ & $.433^{* *}$ \\
\hline $\begin{array}{l}\text { Dominio de herramientas de comunicación } \\
\text { remota (Teams, Skype, Zoom, Weber, } \\
\text { Google Hangout, Google Meet, JitsiMeet }\end{array}$ & $.333^{* *}$ & $.344^{* *}$ & $.337^{* *}$ & $.296^{* *}$ \\
\hline $\begin{array}{l}\text { * p<.05; } \text { p<.05 } \\
\text { Fuente: Elaboración propia }\end{array}$ & & & \\
\hline
\end{tabular}

Resultó interesante que todas las preguntas del cuestionario mostraron una correlación significativa con la satisfacción. No obstante, sobresalen, de forma general, la participación y apoyo de las familias de los/las estudiantes $(r=.400 ; p<.01)$, la preparación para realizar los cambios requeridos $(r=.380 ; p<.01)$, la preparación y disposición para trabajar en colaboración con los/las estudiantes y padres de familia $(r=.400 ; p<.01)$, el apoyo institucional general $(r=.358 ; p<.01)$ y el dominio de herramientas de comunicación remota $(r=.333 ; p<.01)$. Tanto en estas preguntas como en el resto, las correlaciones fueron de carácter positivo, indicando que los niveles de satisfacción aumentan al incrementarse la disposición de dispositivos y recursos tecnológicos, el apoyo familiar e institucional y la preparación y capacitación.

Como parte final del análisis de datos se ejecutaron una serie de regresiones lineales múltiples mediante el método escalonado con la intención de identificar los aspectos que explicaban en mejor medida la satisfacción docente con los resultados obtenidos durante el periodo de confinamiento en cada uno de los niveles educativos. Dado el objetivo planteado, en cada uno de los análisis se consideró a la satisfacción como variable dependiente y al resto de las preguntas del instrumento como independientes. De acuerdo con la Tabla 3, los

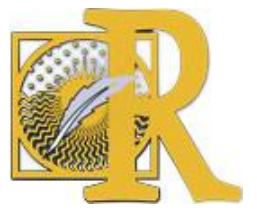

Fecha de recepción: 17-08-2020 Fecha de aceptación: 26-08-2020

Reynoso, O., Portillo, S. \& Castellanos, L. (2021). Modelo explicativo de la satisfacción del profesorado en el periodo de enseñanza remota en México 
tres modelos de regresión obtenidos explican entre el $31.8 \%$ y el $34.0 \%$ de la varianza total, lo cual indica que la magnitud del efecto es entre mediana y grande (Domínguez, 2018).

Tabla 3 - Modelos de regresión por nivel educativo

\begin{tabular}{lccccccc}
\hline \multicolumn{1}{c}{ Modelo } & $\boldsymbol{r}$ & $\boldsymbol{R}^{2}$ & $\boldsymbol{R}^{2}$ ajustado & Error típ. & $\boldsymbol{D W}$ & $\mathbf{f}$ & Sig. \\
\hline Preescolar & .591 & .349 & .338 & .723 & 2.011 & 31.429 & .000 \\
\hline Primaria & .567 & .321 & .318 & .734 & 1.942 & 89.308 & .000 \\
\hline Secundaria & .596 & .356 & .340 & .784 & 2.230 & 23.196 & .000 \\
\hline
\end{tabular}

Nota: $D W=$ Durbin Watson; Variable dependiente: Satisfacción con los resultados escolares obtenidos durante el período de enseñanza remota

Fuente: Elaboración propia

En el caso del nivel preescolar, el modelo quedó constituido por cinco variables exógenas: la participación y apoyo de las familias de los/las estudiantes $(\beta=.283)$, el apoyo institucional global $(\beta=.153)$, la preparación para realizar los cambios requeridos $(\beta=.175)$, el apoyo institucional mediante la elaboración de guías para orientar el trabajo del colectivo docente $(\beta=.156)$ y el dominio de herramientas de comunicación remota $(\beta=.127)$. La tabla 4 muestra los coeficientes estandarizados, no estandarizados y los estadísticos de colinealidad.

Tabla 4 - Coeficientes de regresión de satisfacción docente (Preescolar)

\begin{tabular}{|c|c|c|c|c|c|c|c|}
\hline & \multicolumn{2}{|c|}{$\begin{array}{l}\text { Coeficientes no } \\
\text { estandarizados }\end{array}$} & \multirow{2}{*}{$\begin{array}{c}\text { Coeficientes } \\
\text { estandarizados } \\
\text { Beta }(\beta)\end{array}$} & \multirow[t]{2}{*}{$t$} & \multirow[t]{2}{*}{ Sig. } & \multicolumn{2}{|c|}{$\begin{array}{l}\text { Estadísticas de } \\
\text { colinealidad }\end{array}$} \\
\hline & $B$ & E. std. & & & & Tolerancia & VIF \\
\hline (Constante) & 0.781 & 0.221 & & 3.531 & .000 & & \\
\hline $\begin{array}{l}\text { La participación y apoyo } \\
\text { de las familias de los/las } \\
\text { estudiantes }\end{array}$ & 0.248 & 0.045 & .283 & 5.558 & .000 & 0.856 & 1.169 \\
\hline $\begin{array}{l}\text { Apoyo institucional } \\
\text { global }\end{array}$ & 0.120 & 0.044 & .153 & 2.753 & .006 & 0.724 & 1.382 \\
\hline $\begin{array}{l}\text { Preparación } \\
\text { realizar los cambios } \\
\text { requeridos }\end{array}$ & 0.176 & 0.054 & .175 & 3.290 & .001 & 0.787 & 1.270 \\
\hline $\begin{array}{l}\text { Apoyo institucional } \\
\text { mediante la elaboración } \\
\text { de guías para orientar el } \\
\text { trabajo del colectivo } \\
\text { docente }\end{array}$ & 0.102 & 0.034 & .156 & 2.983 & .003 & 0.809 & 1.236 \\
\hline
\end{tabular}




\begin{tabular}{|c|c|c|c|c|c|c|}
\hline $\begin{array}{lr}\text { Dominio } & \text { de } \\
\text { herramientas } & \text { de } \\
\text { comunicación remota } \\
\text { (Teams, Skype, Zoom, } \\
\text { Weber, } \\
\text { Hangout, Google Meet, } \\
\text { JitsiMeet }\end{array}$ & 0.088 & 0.037 & .127 & 2.338 & .020 & 0.751 \\
\hline
\end{tabular}

Nota: Variable dependiente: Satisfacción con los resultados escolares obtenidos durante el período de enseñanza remota

Fuente: Elaboración propia

El modelo de nivel primaria quedó conformado por siete variables exógenas: la participación y apoyo de las familias de los/las estudiantes $(\beta=.306)$, la preparación para realizar los cambios requeridos $(\beta=.168)$, el apoyo institucional global $(\beta=.107)$, el apoyo institucional mediante dispositivos tecnológicos $(\beta=.093)$, disponer de los dispositivos y recursos tecnológicos necesarios $(\beta=.084)$, el apoyo institucional mediante materiales de trabajo digitalizados/impresos $(\beta=.054)$ y el dominio de herramientas de comunicación remota $(\beta=.054)$. En la tabla 5 se presentan los coeficientes estandarizados, no estandarizados y los estadísticos de colinealidad.

Tabla 5 - Coeficientes de regresión de satisfacción docente (Primaria)

\begin{tabular}{|c|c|c|c|c|c|c|c|}
\hline & \multicolumn{2}{|c|}{$\begin{array}{l}\text { Coeficientes no } \\
\text { estandarizados }\end{array}$} & \multirow{2}{*}{$\begin{array}{c}\text { Coeficientes } \\
\text { estandarizados } \\
\text { Beta }(\beta)\end{array}$} & \multirow[t]{2}{*}{$t$} & \multirow[t]{2}{*}{ Sig. } & \multicolumn{2}{|c|}{$\begin{array}{l}\text { Estadísticas de } \\
\text { colinealidad }\end{array}$} \\
\hline & $B$ & E. std. & & & & Tolerancia & VIF \\
\hline Constante & 0.904 & 0.103 & & 8.771 & .000 & & \\
\hline $\begin{array}{l}\text { La participación y } \\
\text { apoyo de las familias } \\
\text { de los/las estudiantes }\end{array}$ & 0.269 & 0.022 & .306 & 12.405 & .000 & 0.847 & 1.181 \\
\hline $\begin{array}{l}\text { Preparación para } \\
\text { realizar los cambios } \\
\text { requeridos }\end{array}$ & 0.157 & 0.025 & .168 & 6.171 & .000 & 0.692 & 1.445 \\
\hline $\begin{array}{l}\text { Apoyo institucional } \\
\text { global }\end{array}$ & 0.078 & 0.020 & .107 & 3.954 & .000 & 0.704 & 1.420 \\
\hline $\begin{array}{l}\text { Apoyo institucional } \\
\text { mediante dispositivos } \\
\text { tecnológicos }\end{array}$ & 0.060 & 0.016 & .093 & 3.664 & .000 & 0.791 & 1.264 \\
\hline $\begin{array}{lr}\text { Disponer de los } \\
\text { dispositivos } & \text { y } \\
\text { recursos tecnológicos } \\
\text { necesarios }\end{array}$ & 0.069 & 0.021 & .084 & 3.278 & .001 & 0.780 & 1.282 \\
\hline
\end{tabular}

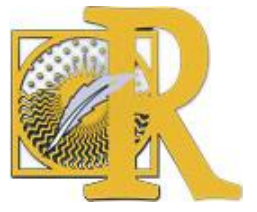


Óscar Reynoso, Samuel Portillo \& Lidia Castellanos. Modelo explicativo de la satisfacción del profesorado en el periodo de enseñanza remota en México

\begin{tabular}{|c|c|c|c|c|c|c|c|}
\hline $\begin{array}{lr}\text { Apoyo } & \text { institucional } \\
\text { mediante } & \text { materiales } \\
\text { de } & \text { trabajo } \\
\text { digitalizados/impresos }\end{array}$ & 0.032 & 0.016 & .054 & 2.048 & .041 & 0.734 & 1.362 \\
\hline $\begin{array}{lr}\text { Dominio } & \text { de } \\
\text { herramientas } & \text { de } \\
\text { comunicación } & \text { remota } \\
\text { (Teams, } & \text { Skype, } \\
\text { Zoom, Weber, Google } \\
\text { Hangout, Google } \\
\text { Meet, JitsiMeet }\end{array}$ & 0.037 & 0.018 & .054 & 2.020 & .044 & 0.726 & 1.377 \\
\hline
\end{tabular}

Nota: Variable dependiente: Satisfacción con los resultados escolares obtenidos durante el período de enseñanza remota

Fuente: Elaboración propia

Por último, el modelo en nivel secundaria se integró por cinco variables independientes: capacitación mediante los directivos de las instituciones $(\beta=.264)$, capacitación mediante cursos y talleres virtuales sobre el uso de tecnología $(\beta=.204)$, preparación y disposición para trabajar en colaboración con los/las estudiantes y padres de familia $(\beta=.166)$, el dominio de herramientas de comunicación remota $(\beta=.139)$ y la participación y apoyo de las familias de los/las estudiantes $(\beta=.117)$. Los coeficientes estandarizados, no estandarizados y los estadísticos de colinealidad del modelo se muestran en la Tabla 6.

Tabla 6 - Coeficientes de regresión de satisfacción docente (Secundaria)

$\begin{array}{lllll}\begin{array}{c}\text { Coeficientes no } \\ \text { estandarizados }\end{array} & \begin{array}{c}\text { Coeficientes } \\ \text { estandarizados }\end{array} & t & \text { Sig. } & \begin{array}{c}\text { Estadísticas de } \\ \text { colinealidad }\end{array}\end{array}$

\begin{tabular}{|c|c|c|c|c|c|c|c|}
\hline & $B$ & E.std. & Beta $(\beta)$ & & & Tolerancia & VIF \\
\hline (Constante) & 0.602 & 0.281 & & 2.141 & .033 & & \\
\hline $\begin{array}{ll}\text { Capacitación } & \\
\text { mediante } & \text { los } \\
\text { directivos de las } \\
\text { instituciones }\end{array}$ & 0.186 & 0.044 & .264 & 4.216 & .000 & 0.782 & 1.278 \\
\hline $\begin{array}{l}\text { Capacitación } \\
\text { mediante cursos y } \\
\text { talleres } \\
\text { sobre el virtuales } \\
\text { tecnología }\end{array}$ & 0.160 & 0.051 & .204 & 3.158 & .002 & 0.736 & 1.360 \\
\hline
\end{tabular}


Óscar Reynoso, Samuel Portillo \& Lidia Castellanos. Modelo explicativo de la satisfacción del profesorado en el periodo de enseñanza remota en México

$\begin{array}{lr}\text { Preparación } & y \\ \text { disposición para } & \\ \text { trabajar } & \text { en } \\ \text { colaboración con } & \text { los/las estudiantes y } \\ \text { padres de familia }\end{array}$

Dominio de

herramientas de

$0.102 \quad 0.044$

.166

2.556

.011

0.723

1.384

comunicación remota

(Teams, Skype,

Zoom, Weber, Google

Hangout, Google

Meet, JitsiMeet

La participación y

0.108

0.054

.117

$2.010 \quad .046$

0.911

1.098

apoyo de las familias

de los/las estudiantes

Nota: Variable dependiente: Satisfacción con los resultados escolares obtenidos durante el período de enseñanza remota

Fuente: Elaboración propia

\section{Discusión.}

El objetivo del presente trabajo consistió en analizar la experiencia de docentes de educación básica sobre algunos aspectos del curso 2019-2020, principalmente, la apreciación sobre la satisfacción con los resultados obtenidos de la enseñanza remota. Tomando en cuenta una serie de aspectos como la disposición de dispositivos y conectividad, el apoyo familiar e institucional en la contingencia y la preparación y capacitación llevada a cabo para enfrentar la situación de emergencia. En tal sentido, mediante un cuestionario, se identificaron los aspectos antes mencionados y se realizó una comparación de acuerdo con el nivel educativo de los docentes. Finalmente, con la intención de reconocer los elementos que explican en mayor medida la satisfacción del profesorado en el periodo de enseñanza remota, se ejecutó un análisis de regresión lineal múltiple en el que se obtuvieron tres modelos, uno para cada nivel educativo.

Comenzando por los hallazgos descriptivos, la satisfacción con los resultados escolares obtenidos durante el período de enseñanza remota, tanto de forma general como en cada nivel educativo, se mostró en un nivel medio, indicando que, aunque hubo valoraciones en los extremos positivos o negativos, la mayor parte del profesorado se mostró medianamente satisfecho con el trabajo efectuado, ello coincide con los resultados reportados por Alves et al., (2020) quienes señalan que en su mayoría los docentes tienen una percepción moderadamente positiva de su estado de bienestar o satisfacción. Una calificación más positiva se presentó al hablar de la disposición de dispositivos y la conectividad para llevar a cabo las clases de forma remota, ya que la gran mayoría de los docentes contaron con los recursos suficientes para efectuar su labor. No obstante, es preciso indicar que hubo algunos profesores que tuvieron muchos inconvenientes en esta área, ya sea por la deficiencia en los

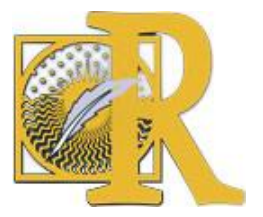

Fecha de recepción: 17-08-2020 Fecha de aceptación: 26-08-2020

Reynoso, O., Portillo, S. \& Castellanos, L. (2021). Modelo explicativo de la satisfacción del profesorado en el periodo de enseñanza remota en México

International Journal of Educational Research and Innovation (IJERI), 15, 229-247

ISSN: 2386-4303 DOI https://doi.org/10.46661/ijeri.5236 
servicios de internet en zonas de difícil acceso o por la ausencia de dispositivos eficientes o actualizados para trabajar a distancia.

La dimensión de apoyo familiar e institucional tuvo bastantes altibajos. De hecho, en el caso del soporte familiar percibido, aunque fue de nivel medio en todos los niveles, los docentes de primaria otorgaron la mejor puntuación, seguidos de cerca por los de preescolar y, con cierta distancia le siguieron los de secundaria. Esta situación podría estar vinculada a los diferentes grados de compromiso y supervisión que el padre de familia debe de prestar en cada nivel. En este sentido, se generó un modelo de regresión por nivel educativo para explicar los elementos relacionados con la satisfacción docente, donde uno de los principales hallazgos hace referencia a una mayor satisfacción del profesorado en los niveles de educación preescolar y primaria en comparación de quienes laboran en educación secundaria, atribuido al aumento en las dificultades (Alves et al., 2020) y a la creciente conflictividad en este tipo de centros (Cantón y Téllez, 2016).

Al revisar el modelo de satisfacción de los docentes de preescolar, la variable exógena que explica una mayor cantidad de varianza es el apoyo de los padres de familia, lo cual manifiesta nuevamente la importancia del involucramiento familiar en el éxito o fracaso de la estrategia de educación a distancia. Lo anterior, resulta lógico si consideramos que la edad del estudiantado que se atiende en este nivel requiere de acompañamiento permanente de un adulto para que exista la posibilidad de considerar el manejo de plataformas y recursos digitales más complejos, así como el cumplimiento de horarios de trabajo y realización de tareas, lo cual coincide con lo señalado por Cifuentes-Faura (2020) quien propone que los padres deben contribuir al desarrollo personal y afectivo del niño, asegurando espacios para el trabajo y diversión, así como otros para los deberes, tareas escolares y ayudar en casa.

En efecto, se acentúa la necesidad del apoyo parental para el cumplimiento de los objetivos de aprendizaje (Cóndor-Herrera, 2020; Morales, 2020). Sobre todo, en una modalidad a distancia y en un contexto de emergencia, donde si bien no se reemplaza al docente, el padre o la madre, han de asumir un rol mucho más activo y participativo en la asesoría y tutoría que requieren los niños y las niñas para el desarrollo de sus tareas de clase; en tanto figuras clave para otorgar al proceso una interactividad, que ayude a la vez a lograr en los más pequeños la motivación necesaria para la realización de las actividades escolares en el hogar, ante un cambio de escenario abrupto, que les ha privado de sus compañeros y compañeras de clase y de las rutinas habituales que tenían lugar en la escuela. En este sentido, la comunicación entre el profesorado y los padres de familia, se constituye como un elemento esencial e indispensable para garantizar la continuidad de la formación de los niños (Cifuentes-Faura, 2020).

Con respecto al modelo correspondiente a docentes de nivel primaria, se mantiene la importancia otorgada por parte del profesorado al apoyo brindado por los padres de familia, al igual que lo ocurrido en el nivel preescolar. Ello resulta comprensible, pues prevalece el envío, recepción, resolución y evidencia de realización de ejercicios escolares. En esta interacción, tal como expresa Cóndor-Herrera (2020) serán los padres quienes apoyen a sus hijos e hijas en el cumplimiento de las actividades. Además, conforme se avance en el nivel de complejidad según la escolaridad, se requerirá de un trabajo conjunto y colaborativo, para posibilitar el logro de los saberes curriculares propios del grado escolar.

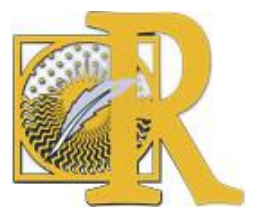

Fecha de recepción: 17-08-2020 Fecha de aceptación: 26-08-2020

Reynoso, O., Portillo, S. \& Castellanos, L. (2021). Modelo explicativo de la satisfacción del profesorado en el periodo de enseñanza remota en México

International Journal of Educational Research and Innovation (IJERI), 15, 229-247

ISSN: 2386-4303 DOI https://doi.org/10.46661/ijeri.5236 
A propósito, algunos estudios han señalado que los padres se involucran en mayor medida en las actividades de los centros educativos cuando sus hijos son pequeños y pareciera que en la medida en que avanza el nivel escolar el involucramiento tiende a ser menor (Stevenson y Baker, 1987, citado por Razeto, 2016). Al mismo tiempo, la participación de las familias en la educación de los hijos, se torna un factor de peso asociado al mejoramiento del rendimiento escolar y a la generación de expectativas positivas ante el profesorado y la institución educativa (Razeto, 2016). Dichos elementos resultan aún más cruciales en una situación de emergencia sanitaria, donde la ausencia presencial del docente multiplica la necesidad de que los padres intervengan con mayor frecuencia en la explicación de contenidos de cierta dificultad a sus hijos, o en la supervisión de las tareas escolares realizadas, en la consulta de dudas a los maestros y maestras, entre otras cuestiones, que habrán de impulsar acciones sinérgicas para apropiación del saber (Morales, 2020).

Además, surge la necesidad de contar con el apoyo institucional, situación que Burgos, Vázquez-Cano, López-Meneses y Adaos (2020) expresan como una declaración que se ha venido implementando de manera positiva para mantener acciones proactivas en cuanto al desarrollo de estrategias educativas. En este sentido, se debe mencionar la necesidad de contar con las condiciones materiales indispensables para desarrollar con éxito la estrategia de enseñanza remota. Ello, con respecto a los dispositivos y conectividad con la que cuenta el profesorado y la que se tiene en el hogar. Sin éstos, sería imposible la localización y uso de materiales y recursos digitales para las clases, el proceso de planeación en sí mismo, el envío de orientaciones, la recepción de las actividades, procesos de retroalimentación y aclaración de dudas, es decir, la posibilidad de una comunicación a distancia entre docentes, estudiantes y padres de familia, como lo demanda la situación actual. Aunque como bien señala Álvarez et al. (2020) se sabe que existen diferencias en infraestructuras, capacidades técnicas y estrategias pedagógicas, el modelo muestra que en la medida en que el docente dispone de las herramientas necesarias para desarrollar la enseñanza a distancia, realiza con mayor entusiasmo su tarea y se siente más satisfecho, contrarrestando la incertidumbre y la desmotivación que suelen acompañar a las circunstancias provocadas por la pandemia. En lo que respecta al tercer modelo, correspondiente al nivel de secundaria, destacan aquellas variables asociadas con la capacitación y la preparación del profesorado, ya sea una capacitación desarrollada por vías institucionales, ofrecida por el centro escolar a sus docentes o una capacitación auto-gestionada por estos últimos. En el primer caso, se trataría de una demostración del respaldo y del interés por parte de la institución escolar y del sistema educativo en su totalidad, hacia los agentes educativos, ante una situación que lo amerita y que podría por tanto explicar la alta valoración de esta variable. En el segundo caso, referente a una capacitación procurada por los mismos docentes, a través de las vías y medios disponibles, habría que considerar una serie de elementos en términos de satisfacción y motivación intrínseca, vinculados al significado que tiene para el profesorado el trabajo que desarrollan, así como la conciencia de la responsabilidad con respecto a su labor social, que les lleva a agenciarse los medios necesarios para mejorar la forma de realizarla (Hackman, 1980, en Galván-Oré, 2008).

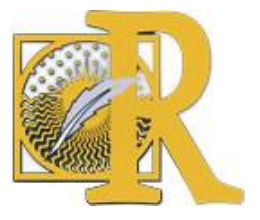

Fecha de recepción: 17-08-2020 Fecha de aceptación: 26-08-2020 Reynoso, O., Portillo, S. \& Castellanos, L. (2021). Modelo explicativo de la satisfacción del profesorado en el periodo de enseñanza remota en México

International Journal of Educational Research and Innovation (IJERI), 15, 229-247 ISSN: 2386-4303 DOI https://doi.org/10.46661/ijeri.5236 
Con respecto a lo anterior, Cóndor-Herrera (2020) menciona la necesidad que tienen los docentes de este nivel por llevar procesos de capacitación y asimilación de la nueva forma de enseñar y aprender, para conocer y desarrollar de mejor manera su rol en este proceso, así como a la necesidad que tiene el docente de percibirse competente para poder realizar la actividad, tal como señalan Franco-López et al. (2019), lo cual en el plano de la satisfacción repercute de manera positiva en mejores resultados y una mayor motivación hacia la clase. Por otra parte, resulta necesario señalar que en este nivel educativo, también se ha mencionado como importante la participación de los padres de familia en los procesos educativos de las niñas, niños y adolescentes. No obstante, en esta ocasión se posiciona en el último criterio que explica la satisfacción del docente a diferencia de lo ocurrido en los niveles de preescolar y primaria que lo posicionan en el primer puesto. Ello se debe al tipo de alumnado que se atiende en educación secundaria, los cuales pueden desarrollar mejor sus actividades de manera autónoma (Cóndor-Herrera, 2020) y requieren menor presencia del padre de familia.

\section{Conclusiones.}

Al momento, se han mostrado múltiples fortalezas y debilidades que presenta el profesorado en torno a la enseñanza remota de emergencia y sus resultados. Por un lado, se destaca la preparación y capacitación efectuada como sinónimo del interés por disponer de las habilidades tecnológicas y pedagógicas para enfrentarse a un nuevo ciclo escolar a distancia y, por otro, se evidencian altibajos en cuestión del apoyo institucional y el involucramiento familiar. En este sentido, los modelos de regresión han permitido distinguir aquellos elementos que influyen en mayor medida sobre la satisfacción del profesorado y el peso que da a cada uno y cómo se modifican conforme se avanza hacia el siguiente nivel educativo. Particularmente, los tres modelos permitieron relacionar la satisfacción del profesorado durante el período de enseñanza remota con factores vinculados a cuestiones de naturaleza social, como, por ejemplo, el sentirse respaldados y apoyados por las familias del alumnado (Cifuentes-Faura, 2020; Cóndor-Herrera, 2020; Morales, 2020), pero también por los directivos y autoridades de las instituciones educativas de pertenencia, elementos señalados por Cantón y Téllez (2016) como los de mayor peso en el estudio de la satisfacción profesional y que integran además, un componente de gratificación determinante hacia la valorización de las tareas educativas (Oros y Main, 2004). A

simismo, sobresalen aspectos vinculados a las condiciones materiales básicas e indispensables para la ejecución exitosa de su rol docente, en una modalidad a distancia que exige contar con una serie de dispositivos y recursos tecnológicos para llevar a cabo el proceso educativo en una coyuntura de emergencia. Ello, hace alusión al carácter organizativo en cuanto a contar con infraestructura, las condiciones necesarias para desarrollar a cabo diversas tareas y el ambiente en el que se desarrollan las mismas (FrancoLópez et al., 2019; Oros y Main, 2004). Por último y no menos importante, encontramos una serie de aspectos que involucran al individuo en sí mismo, a la motivación intrínseca y al compromiso con la labor que desarrolla (Alves et al., 2020; Franco-López et al., 2019; Cladellas-Pros et al., 2018). Por tanto, sentir disfrute y a la vez responsabilidad por las

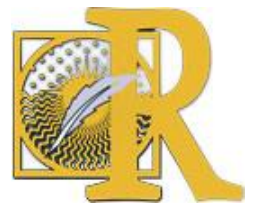

Fecha de recepción: 17-08-2020 Fecha de aceptación: 26-08-2020

Reynoso, O., Portillo, S. \& Castellanos, L. (2021). Modelo explicativo de la satisfacción del profesorado en el periodo de enseñanza remota en México

International Journal of Educational Research and Innovation (IJERI), 15, 229-247

ISSN: 2386-4303 DOI https://doi.org/10.46661/ijeri.5236 
actividades que se realizan evidencia satisfacción, compromiso y una atmósfera positiva de colaboración.

El estudio además hace hincapié en la importancia que el apoyo, involucramiento e implicación familiar tiene en las actividades escolares y en la satisfacción del docente con los resultados, especialmente en los niveles de preescolar y primaria. Si bien este aspecto también es importante en la educación secundaria, en este último nivel escolar, el factor relacionado con la capacitación y preparación del profesorado comienza a tener un peso más determinante en la satisfacción que expresan los encuestados con los resultados.

Finalmente, puede concluirse que, aunque los modelos obtenidos explican de forma retrospectiva los aspectos más importantes para la satisfacción docente, el análisis da pie a advertir el terreno frente a la nueva normalidad y preparase ante un nuevo ciclo escolar en la modalidad remota. En otras palabras, si la participación y apoyo de las familias, las diversas formas de apoyo institucional, la preparación para realizar los cambios requeridos y el dominio de herramientas de comunicación remota son elementos que explican la satisfacción docente, entonces deberán de procurarse y vigilarse en el próximo ciclo escolar en la búsqueda de mejores resultados.

Por tal razón, se valoran las posibilidades y espacios para la capacitación que permita mejorar el desempeño profesional y con ello afrontar de mejor manera una situación imprevista, con los saberes y competencias necesarios para educar en condiciones adversas, diferentes a las tradicionales, bajo un contexto de incertidumbre y presión. Esto invita a desarrollar una comprensión holística de la satisfacción docente, que tome en cuenta los distintos factores asociados para garantizar el desarrollo exitoso de los procesos educativos, aún en un panorama de crisis, y a manera de recomendación, valdría la pena que en estudios posteriores se realice una evaluación más detallada de la satisfacción docente, así como la generación de modelos que integren la perspectiva de otros actores del proceso de enseñanza-aprendizaje, como estudiantes de distintos niveles educativos o los propios padres de familia.

\section{Referencias}

- Álvarez, M., Gardyn, N., lardelevsky, A. y Rebello, G. (2020). Segregación Educativa en Tiempos de Pandemia: Balance de las Acciones Iniciales durante el Aislamiento Social por el Covid-19 en Argentina. Revista Internacional De Educación Para La Justicia Social, 9(3), 25-43. Recuperado de: https://revistas.uam.es/riejs/article/view/12268

- Alves, R., Lopes, T. y Precioso, J. (2020). El bienestar de los docentes en tiempos de la pandemia de Covid-19: factores que explican el bienestar profesional. IJERI: Revista Internacional de Investigación e Innovación Educativa, (15), 203-217. https://doi.org/10.46661/ijeri.5120

- Arce-Peralta, F. (2020). La transición del paradigma educativo hacia nuevos escenarios: COVID-19. CienciAmérica, 9(2), 115-119.http://dx.doi.org/10.33210/ca.v9i2.285

- Archer, N. y De Gracia, G. (2020) Educación superior y covid-19 en la República de Panamá. Revista de Educación Superior en América Latina, 2(8), 15-19. Recuperado de: http://rcientificas.uninorte.edu.co/index.php/esal/article/viewFile/13403/214421444831

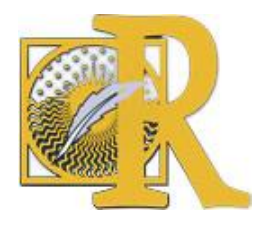

Fecha de recepción: 17-08-2020 Fecha de aceptación: 26-08-2020 
- Arriagada, P. (2020). Pandemia Covid-19: Educación a Distancia. O las Distancias en la Educación. Revista Internacional De Educación Para La Justicia Social, 9(3), 1-3. Recuperado de: https://revistas.uam.es/riejs/article/view/12396

- Bocchio, M. (2020). El Futuro Llegó Hace Rato: Pandemia y Escolaridad Remota en Sectores Populares de Córdoba, Argentina. Revista Internacional De Educación Para La Justicia Social, 9(3), 1-10. Recuperado de: https://revistas.uam.es/riejs/article/view/12473

- Bonilla-Guachamín, J. (2020). Las dos caras de la educación en el COVID-19. CienciAmérica, 9(2), 89-98. http://dx.doi.org/10.33210/ca.v9i2.294

- Burgos, C., Vázquez-Cano, E., López-Meneses, E. y Adaos, R. (2020). DIFPRORET PROJET: Analysis of educational difficulties, proposals and challenges facing the COVID19. IJERI: International Journal of Educational Research and Innovation, 15, 17-34. https://doi.org/10.46661/ijeri.5145

- Caballero, K. (2002) El concepto de "satisfacción en el trabajo" y su proyección en la enseñanza. Profesorado, revista de currículum y formación del profesorado, 6 (1-2), 1-10. Recuperado de: http://www.ugr.es/ recfpro/rev61COL5.pdf

- Cantón, I. y Téllez, S. (2016) La satisfacción laboral y profesional de los profesores. Revista Lasallista de Investigación, 13(1) 214-226. https://doi.org/10.22507/rli.v13n1a18

- Cifuentes-Faura, J. (2020). Consecuencias en los Niños del Cierre de Escuelas por Covid19: El papel del Gobierno, Profesores y Padres. Revista Internacional De Educación Para La Justicia Social, 9(3), 1-12. Recuperado de: https://revistas.uam.es/riejs/article/view/12216

- Cladellas-Pros, R., Castelló-Tarrida, A., y Parrado-Romero, E. (2018). Satisfacción, salud y estrés laboral del profesorado universitario según su situación contractual. Revista de Salud Pública, 20(1), 53-59. https://doi.org/10.15446/rsap.v20n1.53569

- Cóndor-Herrera, O. (2020). Educar en tiempos de COVID-19. CienciAmérica, 9(2), 31-37. https://dx.doi.org/10.33210/ca.v9i2.281

- Domínguez, S. (2018). Magnitud del efecto, una guía rápida. Educación médica, 19(4), 251254.http://dx.doi.org/10.1016/i.edumed.2017.07.002

- Fanelli, A., Marquina, M. y Rabossi, M. (2020) Acción y reacción en época de pandemia: la universidad argentina ante la covid-19. Revista de Educación Superior en América Latina, 2(8), 3-8. Recuperado de: http://rcientificas.uninorte.edu.co/index.php/esal/article/viewFile/13401/214421444829

- Fernández Enguita, M. (2020) Una pandemia imprevisible ha traído la brecha previsible. Recuperado de: https://bit.ly/2VT3kzU

- Figallo, F. (2020) Después de la educación presencial, ¿qué? Revista de Educación Superior en América Latina, 2(8), 41-44. Recuperado de: http://rcientificas.uninorte.edu.co/index.php/esal/article/viewFile/13407/214421444835

- Franco-López, J., López-Arellano, H. y Arango-Botero, D. (2019). La satisfacción de ser docente: un estudio de tipo correlacional. Revista Complutense De Educación, 31(1), 5567. https://doi.org/10.5209/rced.61775

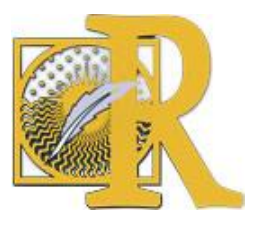

Fecha de recepción: 17-08-2020 Fecha de aceptación: 26-08-2020 
- Galván Oré, L. (2008). Motivación: estrategia de aprendizaje o autorrealización. Revista Digital De Investigación En Docencia Universitaria, 4(1), 1-22. https://doi.org/10.19083/ridu.4.11

- Guerrero, M., Parra, R. y Arce, M. (2018). La satisfacción laboral y su efecto en la satisfacción del cliente, un análisis teórico. INNOVA Research Journal, 3(8), 140-146. https://doi.org/10.33890/innova.v3.n8.2018.879

- Hernández, R. y Mendoza, C. (2018). Metodología de la Investigación: Las rutas cuantitativa, cualitativa y mixta. México: McGraw-Hill.

- Limaymanta-Álvarez, C. (2019). Tendencias en diseños metodológicos en publicaciones indexadas sobre satisfacción laboral de profesores universitarios. RevistaElectrónicaEducare, 23 (3), 1-23. https://doi.org/10.15359/ree.23-3.6

- Locke, E. (1976). The nature and causes of job satisfaction. In M. Dunnette (Ed.), Handbook of industrial and organizational psychology, (pp. 1297-1349). Chicago: Rand McNally \& Co.

- Malo, S., Maldonado-Maldonado, A., Gacel, J. y Marmolejo, F. (2020) Impacto del covid-19 en la educación superior de México. Revista de Educación Superior en América Latina, 2(8), 9-14. Recuperado de: http://rcientificas.uninorte.edu.co/index.php/esal/article/viewFile/13402/214421444830

- Morales, J. (2020). Oportunidad o Crisis Educativa: Reflexiones desde la Psicología para Enfrentar los Procesos de Enseñanza-Aprendizaje en Tiempos de Covid-19. Revista Internacional De Educación Para La Justicia Social, 9(3), 1-9. Recuperado de: https://revistas.uam.es/riejs/article/view/12228

- Muñoz-Chávez, J. y López-Chau, A. (2018). Mobbing y satisfacción laboral en docentes de Instituciones de Educación Superior del centro de México. Una evidencia empírica sobre su relación. Ciencia y Sociedad, 43 (4), 13-21. http://dx.doi.org/10.22206/cys.2018.v43i4.pp13-21

- Murillo, F. y Duk, C. (2020). El Covid-19 y las brechas educativas. Revista Latinoamericana de Educación Inclusiva, 14(1), 11-13. http://dx.doi.org/10.4067/S0718-73782020000100011

- Oros, L. y Main, M. (2004). ESLA-educación: una escala para evaluar la satisfacción laboral de los docentes. RIEE / Revista Internacional De Estudios En Educación, 4(1), 20-34. https://doi.org/10.37354/riee.2004.035

- Pardo, A. y Ruiz, M. (2005). Análisis de datos con SPSS 13 Base. Madrid: McGraw-Hill.

- Pardo, H. y Cobo, C. (2020). Expandir la universidad más allá de la enseñanza remota de emergencia Ideas hacia un modelo híbrido post-pandemia. OutliersSchool. Barcelona.

- Pérez-Narváez, M. y Tufiño, A. (2020). Teleeducación y COVID-19. CienciAmérica, 9(2), 58-64. http://dx.doi.org/10.33210/ca.v9i2.296

- Razeto, A. (2016). El involucramiento de las familias en la educación de los niños. Cuatro reflexiones para fortalecer la relación entre familias y escuelas. Páginas De Educación, 9(2), 190-216. https://doi.org/10.22235/pe.v9i2.1298

- Ruiz, G. (2020). Marcas de la Pandemia: El Derecho a la Educación Afectado. Revista Internacional De Educación Para La Justicia Social, 9(3), 45-59. Recuperado de: https://revistas.uam.es/riejs/article/view/12355

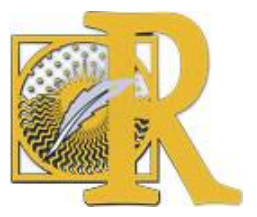

Fecha de recepción: 17-08-2020 Fecha de aceptación: 26-08-2020

Reynoso, O., Portillo, S. \& Castellanos, L. (2021). Modelo explicativo de la satisfacción del profesorado en el periodo de enseñanza remota en México

International Journal of Educational Research and Innovation (IJERI), 15, 229-247 ISSN: 2386-4303 DOI https://doi.org/10.46661/ijeri.5236 
- Sonlleva, M., Martínez, S. y Monjas, R. (2019) Comparación del Grado de Satisfacción del Profesorado de Educación Física con la Formación Inicial y la Inserción Profesional. Revista Iberoamericana de Evaluación Educativa, 12(1), 137-174. https://doi.org/10.15366/riee2019.12.1.009

- UNESCO (2020) Aportes para una respuesta educativa frente al COVID-19 en América Latina. Oficina Regional de Educación para América Latina y el Caribe (OREALC/UNESCO Santiago). Recuperado de: https://unesdoc.unesco.org/ark:/48223/pf0000373761

- Ureña, P. y Castro, C. (2009). Calidad de vida, sentido de coherencia y satisfacción laboral en profesores (as) de colegios técnicos en la Dirección Regional de Heredia. Revista Electrónica Educare, 13(1), 71-87. https://doi.org/10.15359/ree.13-1.6

- Vivanco-Saraguro, Á. (2020). Teleducación en tiempos de COVID-19: brechas de desigualdad. CienciAmérica, 9(2), 166-175. http://dx.doi.org/10.33210/ca.v9i2.307 